\title{
Shrinking Circles: Adaptation to Increased Curvature Gain in Redirected Walking
}

\author{
Luke Bölling, Niklas Stein, Frank Steinicke, Member, IEEE, and Markus Lappe
}

\begin{abstract}
Real walking is the most natural way to locomote in virtual reality (VR), but a confined physical walking space limits its applicability. Redirected walking (RDW) is a collection of techniques to solve this problem. One of these techniques aims to imperceptibly rotate the user's view of the virtual scene in order to steer her along a confined path whilst giving the impression of walking in a straight line in a large virtual space. Measurement of perceptual thresholds for the detection of such a modified curvature gain have indicated a radius that is still larger than most room sizes. Since the brain is an adaptive system and thresholds usually depend on previous stimulations, we tested if prolonged exposure to an immersive virtual environment (IVE) with increased curvature gain produces adaptation to that gain and modifies thresholds such that, over time, larger curvature gains can be applied for RDW. Therefore, participants first completed a measurement of their perceptual threshold for curvature gain. In a second session, the same participants were exposed to an IVE with a constant curvature gain in which they walked between two targets for about 20 minutes. Afterwards, their perceptual thresholds were measured again. The results show that the psychometric curves shifted after the exposure session and perceptual thresholds for increased curvature gain further increased. The increase of the detection threshold suggests that participants adapt to the manipulation and stronger curvature gains can be applied in RDW, and therefore improves its applicability in such situations.
\end{abstract}

Index Terms-Virtual reality, locomotion, redirected walking, psychophysical experiments

\section{INTRODUCTION}

Locomotion is one of the most fundamental spatial behaviors when interacting with the environment. It is our natural way to move from one location to another. Virtual reality (VR) aims to create immersive virtual environments (IVEs) that replicate natural sensation and interaction as closely as possible. Therefore, real walking in IVEs is recognized more and more as an important part of the VR user interface [31]. Natural locomotion interfaces provide more intuitive navigation than, for example, teleportation [15] or walking-in-place gestures [24], and result in increased presence when compared to those forms of transport $[29,36]$. However, the wide-spread use of natural walking as a user interface is compromised by the necessity of a large tracking space in which the user can physically move. Strictly speaking, the size of the virtual space cannot exceed the size of the physical space.

Redirected walking (RDW) is a set of techniques to extend the borders of the virtual space beyond the limitations of the physical space while still allowing almost natural walking [23,33]. In RDW, the user views the virtual scene with a head-mounted display (HMD). The position and orientation of the HMD is tracked and the user's view of the scene is updated accordingly. Hence, the user can freely walk in physical space while experiencing an IVE. Unbeknownst to the user, however, the position and orientation updates are slightly modified so that the user is visually guided along a physical path that may differ from the virtual path and remains physically within the available tracking space.

To maintain a user's high sense of presence in the IVE the deviations imposed by RDW should ideally remain imperceptible to the user. In particular, previous research on detection thresholds for cur-

- Luke Bölling is with the University of Muenster. E-mail: l.boelling@uni-muenster.de.

- Niklas Stein is with the University of Muenster. E-mail: niklas.stein@uni-muenster.de.

- Frank Steinicke is with the University of Hamburg. E-mail: steinicke@informatik.uni-hamburg.de.

- Markus Lappe is with the University of Muenster. E-mail: mlappe@uni-muenster.de.

Manuscript received xx xxx. 201x; accepted xx xxx. 201x. Date of Publication xx xxx. 201x; date of current version xx xxx. 201x. For information on obtaining reprints of this article, please send e-mail to: reprints@ieee.org. Digital Object Identifier: $x x . x x x x / T V C G .201 x . x x x x x x x$ vature gains, i.e., gains that modify the curvature of the user's path during walking, shows that walking in a physical circle with a radius of about $20 \mathrm{~m}$ is indistinguishable from walking in a straight line [30]. Thus, a tracked area of about 40 by $40 \mathrm{~m}$ is required. Recent work has shown that the space requirement can be decreased with improved HMDs [8], with specially designed mappings between physical and virtual space [14,35], or by leveraging perceptual limitations introduced by eye movements $[2,17,34]$. While such methods relax the space requirements of RDW to some degree $[1,11,19]$, the necessary physical space is still larger than tracking areas commonly available, or than the Room-Scale VR targeted by the gaming and entertainment industries. Thus, additional methods to decrease the necessary tracking space are highly desirable.

This paper aims at this issue from the viewpoint of the user's perceptive state in the IVE. Our sensory experiences and expectations are shaped by our sensorimotor interactions with the world. The fact that we receive a certain visual feedback when we act, for instance, a certain retinal motion of the environment when we walk forward, is part of an action-perception loop that links our actions with their sensory consequences $[7,22]$. Actions, in turn, generate predictions for sensory consequences. When the sensory input does not match these predictions, for example, when a strong curvature gain is introduced, the mismatch becomes noticeable [30].

However, when such a mismatch persists over some time, the brain adapts to the changes in sensorimotor contingencies and re-calibrates the sensorimotor loop. This adaptive property of the perceptual system opens possibilities for additional manipulation. If the adapted gain becomes the new sensorimotor expectation of the system, and deviations are detected only if they are sufficiently different from the adapted gain, adapting the user to a new normal gain should produce elevated thresholds for curvature detection. This would allow RDW algorithms to use stronger curvature gains without users noticing and therefore, with reduced risk of a break in their sense of presence. In the present paper we test this idea for its applicability in redirected walking.

The remainder of the paper is structured as follows. Section 2 reviews literature related to our work, in particular, to sensorimotor adaptation and redirected walking. Section 3 describes the experiments that we conducted to analyze the adaption to RDW. In Section 4, we describe and discuss the results. Section 5 shows how the findings can be applied to curvature gain. Section 6 presents a general discussion. Section 7 concludes the paper. 


\section{Previous Related Work}

In this section we first provide background information on sensorimotor adaptation and re-calibration. Next, we will describe how locomotion is performed in VR and, in particular, how redirected walking techniques work.

\subsection{Sensorimotor adaptation}

The human visual system is extraordinarily plastic when confronted with changes in the relationship between the self and the world. One of the most dramatic illustrations of this plasticity are the experiments with inversion glasses in the early 20th century. These experiments have shown that the brain can adapt over time to a complete inversion of the visual field and can learn to perform adequate actions, including walking and cycling, using the upside-down input characteristics. More subtle modifications of sensorimotor contingencies and associated perceptual changes have been extensively researched in the paradigm of prism adaptation (reviewed in [25]), which bears some resemblance to RDW techniques. In prism adaptation, the subject wears a set of prismatic glasses that displace the view of the world off to the side by a small angle, say 10 degrees. In consequence, actions such as reaching for a visual target initially miss the target by about the same angle. Over several repetitions of the action, the subject gradually adapts her motor control to successfully prepare reaching movements for the displaced target. This adapted sensorimotor program becomes the new normal state of sensorimotor control for this environment Prism adaptation also influences perception such that the subjective straight ahead is shifted and subjects perceive the axis of their body somewhat rotated [25].

Wearing prisms while walking produces curved trajectories much like redirected walking does $[18,26]$. Bruggeman et al. [6] and Saunders and Durgin [27] replicated this effect in VR and investigated adaptation to a constant offset between physical and virtual directions of heading during walking. They found that a displacement of 10 degrees between physical and virtual heading was imperceptible to participants and that participants walked in a curve when they attempted to walk a straight line to the target. After a small number of trials with this offset, participants partially compensated for the offset and produced straighter walking paths. Visual feedback from the environment during walking, in particular optic flow, was a major factor in producing the adaptation.

Subsequent studies also tested whether perception was affected by the displacement between physical and virtual heading during walking In a HMD study, Bruggemann and Warren [5] found that adaptation was specific to locomotion, but did not transfer to other tasks such as throwing or kicking. Herlihey and Rushton [9], on the other hand, found that walking with prism glasses produced a change in the perceived straight ahead direction of egocentric space. One of the possible reasons of the different results of these studies might be the differences between prism glasses and HMDs [9]. In contrast to the previous work, this paper focusses on possible changes in perceptual detection thresholds for the incongruency between physical and virtual walking, which has not been studied so far.

\subsection{Redirected walking}

Since its introduction in 2001 [23] RDW has been an active field of research in VR. Early work and recent advances have been summarized in several review articles $[4,16,21]$. A taxonomy of gain manipulations for RDW has been described by Suma et al. [33]. It comprises rotation gains that affect the user's direction during rotations, translation gains that affect the user's displacement during forward motion, and curvature gains that affect the curvature of the user's path during walking. Rotation and translation gains are implemented as scaling factors by which any rotation or translation of the user is scaled when rendering the view in the HMD. For example, a rotation gain of $G_{R}=1.1$ means that the rotation of the view of the scene in the HMD is $1.1 \cdot \alpha$, where $\alpha$ is the angle that the user physically rotates. Likewise, any physical translation $T$ of the user is multiplied by the translational gain $G_{T}$ before the view is rendered in the HMD. Curvature gains, which are the focus of the present paper, describe differences between the curvature of the physical path that the user walks in the tracking space and the

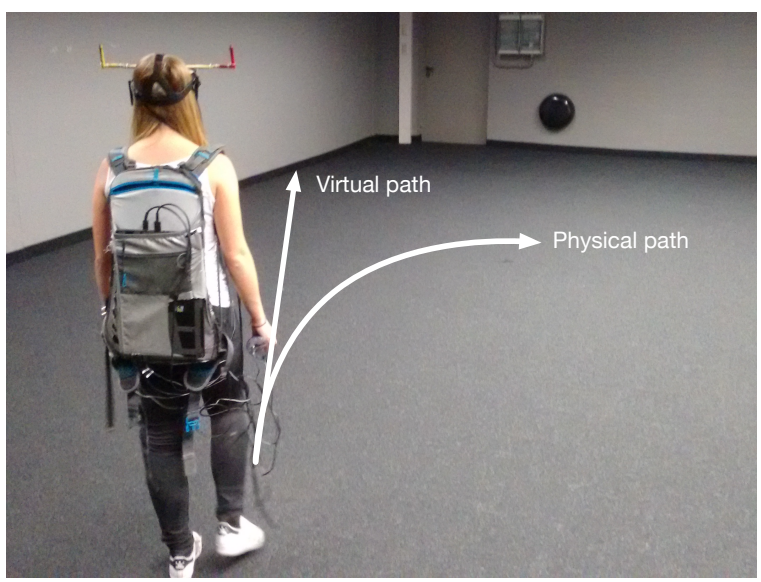

Fig. 1. Redirected walking based on curvature gain manipulation. The user wears a HMD connected to computer in a backpack, and is free to walk in the lab. Her head position and orientation is tracked with an optical tracking system and an gyroscopic orientation sensor. The view of the virtual scene is manipulated such that the user is guided along a physical path that is more curved than the path experienced in the IVE.

virtual paths that the user experiences in the virtual environment. The curvature gain $G_{C}:=\pi / r$ can be defined via the inverse of the radius $r$ of the physical path onto which user is redirected while walking a straight path in the IVE [30]. Table 1 shows the curvature gains and corresponding radii used in the present study. In order to make the user walk on the curved physical path the scene is rotated in the HMD depending on the user's position on the path with a velocity that is linked to the physical forward velocity of the user during walking.

Steinicke et al. [30] measured detection thresholds for such curvature gains by asking participants to discriminate between leftward and rightward physical paths with different curvature gains. They found that $75 \%$ detection thresholds were $G_{C}=\pi / 69 m^{-1}$. At these thresholds, users were physically walking on a circle of radius of about $22 \mathrm{~m}$ without noticing the difference to a straight path. This corresponds to unnoticeable rotations of about 2.6 degrees per meter. Later research extended these results and showed that thresholds depend, for example, on forward velocity or the layout of the environment, and that the radius can come down to less than $10 \mathrm{~m}$ in some conditions $[3,8,10,12,14,20]$.

Besides gain manipulation during walking, several other techniques have been introduced to further improve RDW and reduce the required size of the tracking and walking area. These include manipulations of the orientation of the view during change blindness [32], during saccadic eye movements [2,34], or during eye blinks [17]. In each of these cases, the view in the HMD can be subtly rotated without the user's notice. Since these techniques can be applied even when the user is standing still, they provide an important set of tools to expand the applicability of RDW and to increase its effectiveness. However, current eye tracking capabilities in VR are still limited by the low sample rates of low-cost eye trackers nowadays often used in HMDs. For example, Bolte and Lappe [2] had to build a custom electrooculography system into their HMD setup to sample eye position with sufficient temporal resolution to detect saccades, while Sun et al. [34] had to use a high-end eye tracker built into a VIVE HMD that is 10 times more expensive then current consumer oriented solutions such as the Fove ${ }^{1}$. Hence, the focus of this works is to identify alternatives to increase the efficiency of gains.

\section{Experimental Adaptation of Curvature Gain}

The aim of the experiment was to measure how much users adapt to a constant unidirectional curvature gain and whether this adaptation produces changes in perceptual threshold for the differences between physical and virtual paths. Adaption requires that the user stays and

\footnotetext{
${ }^{1}$ www.getfove.com
} 


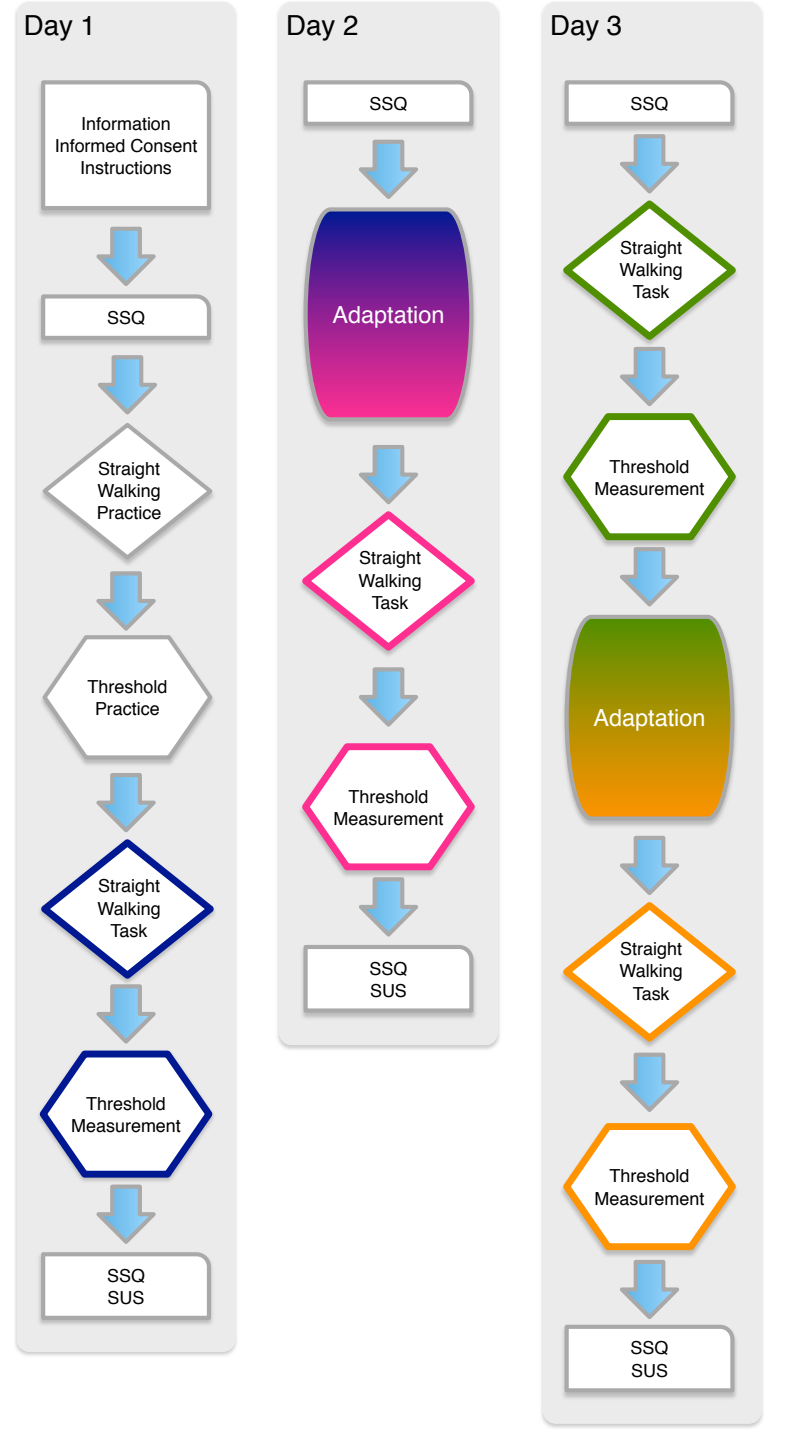

Fig. 2. Sequence of sessions during the three days of the experiment. On day 1 participants were instructed about the experiment and the tasks, gave informed consent, and filled out a pre-experiment simulator sickness questionnaire (SSQ). Then, participants were allowed practice blocks of the straight walking task and the threshold measurement task to familiarize themselves with the tasks and the IVE. Afterwards, baseline measurements were taken in the two tasks (blue/bold boxes). Finally, participants completed the SSQ again as well as the Slater-Usoh-Steed presence questionnaire (SUS). On day 2, after filling out the SSQ, participants were exposed to the adaptation block, which consisted of 150 walks with a constant curvature gain of $G_{C}=+\pi / 30 \mathrm{~m}^{-1}$. Thereafter post-adaptation measurements (magenta/bold boxes) were taken for both tasks, followed by a post-experiment SSQ and SUS. Day 3 started with a pre-experiment SSQ and a re-test of the straight walking task and of the threshold measurement (green/bold boxes). Then, participants completed another adaptation block of 150 trials before they were tested for further adaptation in the straight walking task and the threshold measurement (orange/bold boxes). The experiment ended with the post-experiment SSQ and SUS.

acts within the IVE for a certain time and learns to interact with the IVE in a particular way. Therefore, we asked our users to perform repeated walks between targets for about 20 minutes with a single fixed rightward curvature gain. Measurements of walking behaviour and perceptual thresholds were taken after such adaptation and compared to baseline measures taken before adaptation. The experimental procedures are described in detail in the following sections.
Table 1. Curvature gains and radii used in this study.

\begin{tabular}{cccccccc}
\hline$G_{C} / m^{-1}$ & $-\pi / 15$ & $-\pi / 30$ & $-\pi / 60$ & 0 & $\pi / 60$ & $\pi / 30$ & $\pi / 15$ \\
\hline radius $/ m$ & -4.8 & -9.6 & -19 & $\infty$ & 19 & 9.6 & 4.8 \\
\hline
\end{tabular}

\subsection{Setup}

The experiments were conducted in a walking arena of $10 \mathrm{~m} \times 7 \mathrm{~m}$. The participant wore an Oculus Rift CV1 HMD with a nominal field of view of 110 degs. and a resolution of 1080 x 1200 pixels per eye. The view of the scene was presented in the HMD depending on head position and orientation. The scene depicted a desert environment that was designed to be natural and detailed to increase immersion but at the same time somewhat generic and without highly conspicuous landmarks, so that rotational changes during the threshold measurements were not disturbing.

Head position was tracked with an active optical tracking system (Precise Position Tracking manufactured by WorldViz) with an update rate of $90 \mathrm{~Hz}$. Head orientation was tracked with the gyroscopic sensors of the HMD and was realigned with the optical tracking system after every trial. Stimuli were presented with a MSI GE63VR 7RF Raider notebook and presented with Unity 2017.4.3f1. User input was recorded with a Macally Airstick, that was equipped with an LED so that it could be tracked with the optical tracking system. The participants wore a backpack, in which the notebook and two batteries were carried.

\subsection{Participants}

Twelve participants ( 8 male, 4 female) took part in the study and completed the full set of sessions. Participants were student or members of the Department of Psychology and Sports Science of the University of Muenster, ranging in age from 20 to 38 years (mean age 25 years). All participants had normal or corrected-to-normal vision and no history of visual or motor problems. The gave written informed consent to the participation on the study. Experimental procedures were approved by the Ethics Committee of the Department of Psychology and Sports Science of the University of Muenster.

\subsection{General Procedures}

The experiment consisted of several phases that were intended to measure baseline performance, adaptation effect, retention, and further adaptation, and were conducted on three successive days. On the first day, participants were familiarized with the tasks and pre-adaptation threshold measurement were taken. These consisted of a straight walking task, in which the subject was simply asked to walk straight towards a memorized target, and a measurement of thresholds for perception of curvature gain. Each of these blocks was run twice. The first blocks were considered as a practice block to familiarize the participant with the setup and the measurement procedures. Data from these blocks was not included in the analysis. The second blocks were taken as the baseline measure with which subsequent post-adaptation measurements were compared.

The adaptation itself took place on day 2 followed by post-adaptation walking and threshold measurements. Effects of adaptation on the threshold and straight walking tasks are expected in a comparison of the post-adaptation measurements to the baseline measurements.

Day 3 started with a re-test of straight walking and threshold measurement to test potential retention of adaptation from day 2, followed by another adaptation session and a final walking and threshold measurement to assess any further adaptation effects. Possible retention of adaptation on day 3 would be shown in a comparison between re-test data and baseline data from day 1 . A comparison of the final measurements on day 3 to the measurements after initial adaptation on day 2 would show whether adaptation effects increased after a further adaptation session.

The full sequence of events during the three days of testing is depicted in Figure 2. Total duration of testing on each day was about one hour. The detailed procedures for threshold measurements, adaptation, and straight walking are described below. 

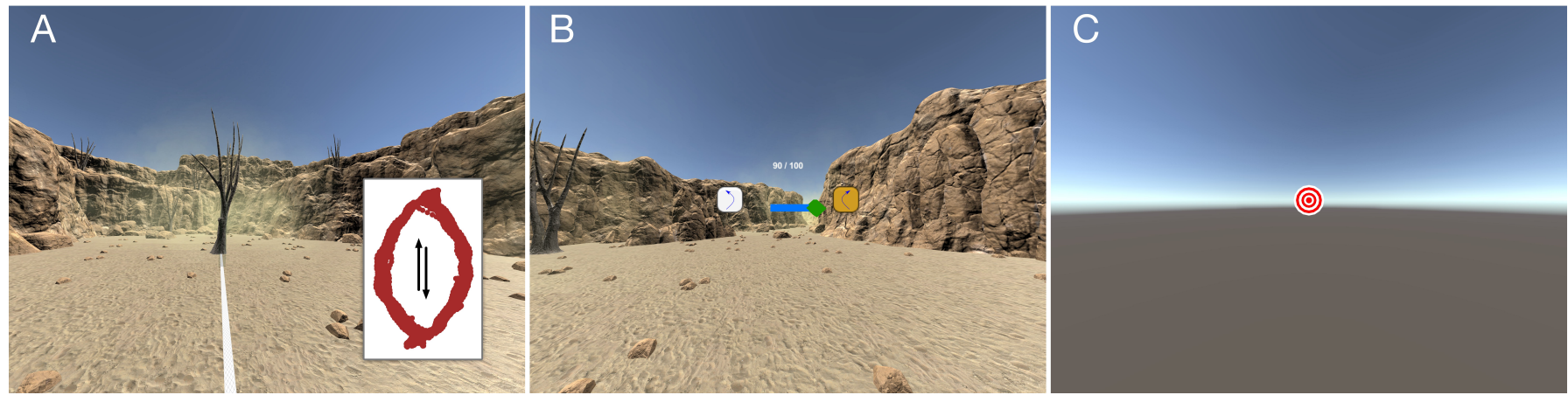

Fig. 3. A: Depiction of the virtual environment visible to the user in the adaptation and threshold measurement blocks. The scene consisted of an outdoor desert environment. Walking tracks were present on the ground. The participant walked virtually along the straight track while a curvature gain was imposed to lead her along a curved path in physical space. The inset shows physical walking paths of 150 back and forth walks during one example adaptation session. Physical paths (red) were always curved rightward while virtual paths for both ways (black arrows) were always straight. $\mathrm{B}$ : In the threshold measurements, participants had to indicate whether the physical path that they had walked was curved to the left or to the right. The two response option were depicted graphically on the screen in front of the participant and the participant responded by selecting one of the two alternatives through button press. C: The virtual view during the straight walking task. The scene was removed and the participant saw only blue sky above the horizon together with a bullseye target. The participant pointed towards the target and pressed a button to start the trial. Then, the target was extinguished and the participant had to walk straight in the direction of the target.

\subsection{Threshold Measurements}

The threshold measurements tested changes in perceptual detection of curvature gains following adaptation. The procedures for threshold measurements followed those of Steinicke et al. [30] and used a standard psychophysical discrimination method based on the 2AFC task Participants were instructed to walk along a straight line in the virtual environment. The scene presented a straight pathway on the ground in a desert scene which the participants had to follow (Figure 3A). In different trials, curvature gains of different magnitudes and directions were imposed such that participants physically had to walk along different curved paths in order for the virtual path to stay straight. After each trial, participants were asked to choose between two response alternatives, i. e. whether their physical walking path was curved leftward or rightward (Figure 3B). They had to decide between these two possibilities even when they felt unsure about the correct answer. They responded by pressing the $\mathrm{C}$ (for left) or $\mathrm{B}$ (for right) button on the controller. The answer was confirmed with the A button. The responses were recorded and used to estimate psychometric functions for the percentage of correct responses.

We used seven different values of curvature gain (Table 1) in a constant stimuli paradigm with 9 repetitions for each gain resulting in 63 trials in total for each measurement. The order of stimuli was randomized and counterbalanced. The straight distance from the start to the end of the walking path was $6 \mathrm{~m}$. Curvature gains were applied with the onset of the walk. Participants were instructed to walk at a natural pace and to stay as close as possible to the visible path.

\subsection{Adaptation Procedures}

In the adaptation phase, participants were required to walk between two targets while a constant curvature gain of $G_{C}=+\pi / 30 \mathrm{~m}^{-1}$ was applied. We specially choose this gain as rounded value of the detection thresholds described in [30], such that the manipulation is not noticeable to the participants, but still provides an ecologically valuable amount of RDW manipulation [3,14, 16, 20,30]. The scene depicted the same outdoor desert landscape as in the threshold measurements (cf. Figure 3A). Participants started on one side of the tracking arena with orientation towards the opposite side. The target was placed $6 \mathrm{~m}$ in front of the participant along with a straight path as in the threshold measurements. The participant was instructed to walk directly to the target along the indicated path. Curvature gain was applied during the walking and led to a physical path that was curved but that ensured that the participant ended in the target location on the opposite side of the tracking arena. When the participant reached the target location, she was instructed to turn around to face the next target, which was placed on the opposite location of the tracking arena. Then, the participant walked to that target while the same curvature gain was applied. This was repeated for a total of 150 walks which lasted about 20 minutes.

\subsection{Straight Walking Task}

The straight walking task was included to measure changes in motor control after adaptation. In the straight walking task, the visual structure of the scene was removed such that the screen depicted only a horizon and clear sky (see Figure 3C). Therefore, the participant received no visual feedback about the speed and direction of walking. If participants adapted their walking control to the changed gain they would be expected to walk on a circular path towards a straight ahead location even without manipulated visual feedback.

At the beginning of each straight walking trial, participants saw a bullseye target $8 \mathrm{~m}$ away in mid air at a height of $1.70 \mathrm{~m}$ above the ground (Figure 3C). Participants turned to orient themselves to point directly towards the target and pressed a button. Then, the target was removed and participants had to walk straight into the target's direction. Head position during the walk was recorded and stored for later analysis. This data was used to measure changes in motor control by analyzing the walking path taken by the participant. After the participants had walked for $5.75 \mathrm{~m}$, a head-fixed arrow appeared to indicate that the participant needed to stop, turn around, and walk back to the starting location for the next trial which was indicated by a red transparent pillar. Each straight walking block consisted of 5 trials.

\subsection{Questionaires}

Before and after each session participants filled out the Simulator Sickness Questionnaire (SSQ) [13]. After each session participants filled out the Slater-Usoh-Steed (SUS) presence questionnaire [37].

\subsection{Data Analysis}

For the straight walking task, head positions of the participants during walking were averaged across trials in each condition. Walking paths for each subject were constructed by taking a sliding mean over the data points along the direction towards the target (z-axis) with a width of $1 \mathrm{~m}$ to remove high frequency variations of the head position to head bobbing and sway during walking. Statistical tests for differences between conditions were were carried out on the difference between start and end points of the walking trajectories in $x$ (sideways) direction.

For the threshold measurements, logistic psychometric curves of the form

$$
S(x ; m ; w)=\frac{1}{1+e^{-2 \log \left(\frac{1}{0.05}-1\right) \frac{x-m}{w}}}
$$




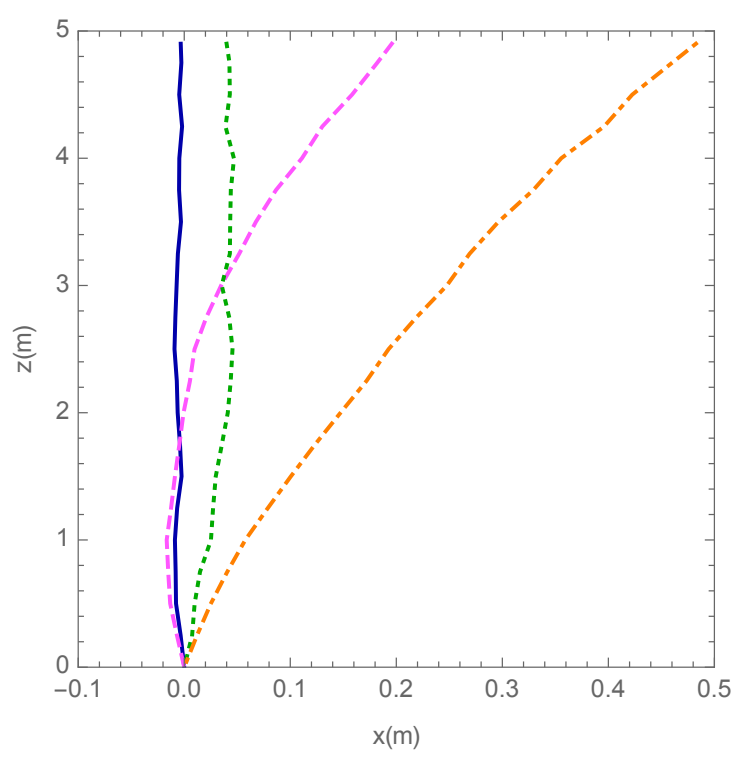

Fig. 4. Walking trajectories in the straight walking task before and after exposure to a constant curvature gain of $G_{C}=+\pi / 30 \mathrm{~m}^{-1}$. Colors correspond to conditions described in Fig. 2. In the baseline measurements before adaptation (blue line), participants walked a straight path towards the target. After adaptation (magenta dashed line) the path taken by the participants is curved and deviates to the right from the straight line to the target, showing the influence of adaptation on motor behavior. In the re-test on day 3 (green dotted line) participants again walked straight to the target, but with a small rightward deviation. After further adaptation on day 3 (orange dot-dashed line) the path again deviates to the right.

were fitted to the data of each condition using the psignifit4 package for Matlab [28]. In this function, $m$ provides the mean and $w$ provides the width. From these, the point of subjective equality (PSE) and the slope were calculated, which were used for statistical testing. Statistical testing was done with $\mathrm{R}$ version 3.4.4.

\section{Results}

We measured changes in motor control and detection of curvature gain after adaptation to a constant curvature gain of $G_{C}=+\pi / 30 m^{-1}$. We begin by describing changes in motor control before we describe changes in detectability of curvature gains.

\subsection{Straight Walking Task}

In the straight walking task participants saw a target in an otherwise unstructured scene. The target was then removed from view and participants were asked to walk straight to the remembered target location.

A Friedman test on the sideways deviation per trial per subject of the walking trajectories showed a significant effect of condition $\left(\chi^{2}=13.3, \mathrm{df}=3, p=0.004\right)$. Post-hoc holm-corrected Wilcoxon tests (All: $p<0.001$ ) revealed that the deviation after adaptation on day 2 was significantly different from the baseline taken on day 1 and that the deviation after adaptation on day 3 was significantly different from the re-test trajectory on the start of day 3 . There was also a significantly difference between the re-test on day 3 and the baseline of day 1 .

Figure 4 shows the averaged walking trajectories before and after adaptation. Color codes match those in Figure 2. The blue line shows data from the pre-adaptation baseline session that was obtained prior to any adaptation or threshold measurements on day 1. Participants walked in a straight line towards the target as pointed out in the instruction. After the first adaptation session on day 2 (magenta line), participants deviated from the straight course and produced a curved path in the direction of the path curvature enforced by the curvature gain in the adaptation block. Note that in the straight walking task no curvature gain was imposed and the virtual scene did not provide any visual feedback about imposed gains or speed and direction of walking. Therefore, the path taken by the participants clearly shows a change in motor behavior that was produced by the adaptation block. The green line shows results from the straight walking task performed at the beginning of the third day. Participants at that time produced much straighter walking trajectories than after the adaptation on day 2. However, the average walking trajectory was still tilted a bit to the right compared to the baseline trajectory measured before adaptation. After the adaptation block on day 3 (orange line) participants showed a strong deviation of their walking path, confirming that changes of motor behavior occurred during adaptation.

\subsection{Threshold measurements}

Response data from the threshold measurements were fitted with logistic psychometric functions using the psignifit4 package for Matlab [28]. Responses from two participants indicated that they did not perform the task or did not follow instructions. The data from these participants showed inverted psychometric functions so that they reported rightward more often when the display presented leftward curves and vice versa. It is likely that these participants misunderstood the task instructions or confused the response procedure so that they mixed-up the two responses. Their data could not be fitted with the regular psychometric function and hence were excluded from the analysis. From the fits of the remaining participants the point of subjective equality (PSE) was determined in each condition to measure shifts of the psychometric curve induced by adaptation. The point-of-subjective equality (PSE) is the gain at which participants perceive their physical path as straight or normal and for which they cannot decide whether the curvature was leftward or rightward. In this case, they have to guess the direction of curvature and report rightward and leftward gain each with a chance probability of $50 \%$.

A Friedman test on the PSE with condition as a factor showed a significant effect of condition $\left(\chi^{2}=14.52, \mathrm{df}=3, p=0.002\right)$. Post-hoc holm-corrected Wilcoxon tests revealed that the PSE after adaptation on day 2 was significantly different from the baseline on day 1 ( $p=$ 0.012 ) and that the the PSE after adaptation on day 3 was significantly different from the re-test at the start of day $3(p=0.049)$ and from the baseline taken on day $1(p=0.049)$. Thus, the adaptation shifted the psychometric function. The re-test PSE on day 3 was also significantly different from the PSE after adaptation on day $2(p=0.049)$ but not from the baseline taken on day 1 .

To estimate any change in the steepness of the psychometric function between conditions we calculated the slope at the $50 \%$ point. A Friedman test on the slope with condition as a factor showed no significant effect of condition $\left(\chi^{2}=3.72\right.$, $\left.\mathrm{df}=3, p=0.293\right)$. Thus, the adaptation did not affect the steepness of the psychometric function.

Figure 5 shows the grand average psychometric functions obtained from collapsing the data from all subjects in each condition. The blue line shows the psychometric curve obtained on the first day prior to the adaptation, i.e. the baseline measurement. In the baseline condition, the PSE is at $\pi / 305 \mathrm{~m}^{-1}$, very close to zero, and corresponding to a radius of $97 \mathrm{~m}$. Thresholds for reliable detection of curvature gains are commonly defined as the gain values for which the participants reach $75 \%$ correct responses [30]. These are the $75 \%$ points on the curve for rightward gain and the $25 \%$ point for leftward gain. In the baseline condition, these values are $\pi / 24 m^{-1}$ and $-\pi / 35 m^{-1}$, corresponding to radii of $7.7 \mathrm{~m}$ and $11 \mathrm{~m}$. These thresholds are higher than those reported by Steinicke et al. [30], but consistent with some of the ranges reported in later studies $[3,8,10,12,14,16,20]$. As suggested by these authors, thresholds may vary between studies depending on the virtual environment, the larger field of view of todays HMDs, or the participants. For the present study, the absolute values of the thresholds are less important since we are interested in changes of thresholds produced by adaptation.

After exposure to a constant curvature gain of $G_{C}=+\pi / 30 m^{-1}$ in the first adaptation session on day 2 (magenta line in Figure 5) the psychometric curve is shifted to the right. The PSE is now at $\pi / 38 m^{-1}$, corresponding to a radius of $12 \mathrm{~m}$. Thus, a curved path with a radius of $12 \mathrm{~m}$ is now perceived as straight or normal. Hence, the adaptation changes the path that appeared straight to the participants from $97 \mathrm{~m}$ 
radius to $12 \mathrm{~m}$ radius. Therefore, perception of curvature gain was changed by the adaptation block.

The shift of the psychometric curve also affected thresholds for detection of curvature gain. The detection threshold for rightward curvature gain ( $75 \%$ point on the curve) changed from $\pi / 24 m^{-1}$ to $\pi / 17 m^{-1}$, corresponding to a radius of $5.4 \mathrm{~m}$. Therefore, after adaptation curvature gains that were reliably detected in the baseline measurement were no longer detectable. Thus, after adaptation higher curvature gains can be applied without the participant noticing. For the $25 \%$ point, on the other hand, i. e., the detection threshold for leftward curvature gain, the shift of the psychometric curve to the right leads to enhanced detectability of leftward curvature gains. The threshold changes from $-\pi / 35 m^{-1}$ (radius $11 \mathrm{~m}$ ) before adaptation to $-\pi / 70 m^{-1}$ (radius $22 \mathrm{~m}$ ) after adaptation, meaning that curvature gains that were not noticeable before adaptation became detectable after adaptation.

The green line plots the psychometric function measured at re-test on the start of the third day. The curve is shifted back towards the baseline obtained before adaptation on day 1 . The PSE is $\pi / 850 m^{-1}$ (radius $270 \mathrm{~m}$ ). The adaptation session on the third day (orange line) then produced about the same PSE as the adaptation on day two: $\pi / 44 m^{-1}$, radius $14 \mathrm{~m}$.

The $75 \%$ detection thresholds remained elevated on day 3 . In the retest measure (green), the threshold was at $\pi / 17 m^{-1}$ (radius $5.5 \mathrm{~m}$ ), similar to the value after adaptation on day 2 . After the second adaptation session it remained at a similar value $\left(\pi / 18 \mathrm{~m}^{-1}\right.$, radius $\left.5.9 \mathrm{~m}\right)$. The $25 \%$ thresholds were $-\pi / 37 m^{-1}$ (radius $12 \mathrm{~m}$ ) at re-test and $-\pi / 11 \mathrm{~m}^{-1}$ (radius $36 \mathrm{~m}$ ) after the further adaptation.

\subsection{Questionaires}

On each day, participants completed the SSQ before and after all sessions. The mean pre-post difference in SSQ score was largest on the first day $(13.3, S D=1.7)$ and lower on the following days (day 2: 5.0, $S D=1.8$; day 3: $7.8 S D=1.8$ ). This shows that simulator sickness became smaller as participants became more experienced with the IVE and the gain manipulation.

The mean SUS score for the sense of feeling present in the IVE was $4.1(S D=1.8)$ on a seven-point Likert scale, and did not change over the course of adaptation (day 1: $4.3, S D=1.7$; day 2: $4.0, S D=1.8$; day 3: $4.1, S D=1.8$ ).

\subsection{Discussion}

Our results show that both motor behavior and perceptual thresholds were affected by exposure to a constant curvature gain of $G_{C}=$ $+\pi / 30 \mathrm{~m}^{-1}$ during a $20 \mathrm{~min}$ session of 150 trials of walking in VR. The straight walking test showed that after adaptation participants walked on a circular path even in an unstructured scene and without manipulated feedback. This is similar to observations after prism adaptation and prism-like constant offset conditions in VR [6, 18,26,27]. However, it has not been tested before for curvature gain manipulation as used in RDW.

The change of perceptual thresholds is also a novel finding for curvature gain detection and has implications for RDW, as discussed in the next section. The re-testing on day three of our measurement protocol showed that motor behavior and perceptual thresholds mostly shifted back towards the pre-adaptation state, although a small significant effect on walking trajectories persisted. The decrease of adaptation presumably involves sensorimotor interactions with the real world in which no curvature gain is applied. The measurements on day 3 further showed that users will re-adapt motor behavior and perceptual thresholds when exposed to the constant curvature gain of $G_{C}=+\pi / 30 m^{-1}$ again. Taken together, the results show that both motor behavior and perception are plastic and will adapt in IVEs in which sensorimotor gain is manipulated.

\section{Implications FOR REDIRECTED WALKING}

Curvature gain manipulation in redirected walking aims at producing physical walking paths that are more curved than virtual walking paths in order to keep the user in a confined tracking space. Ideally, such manipulations are performed without the user noticing any discrepancy
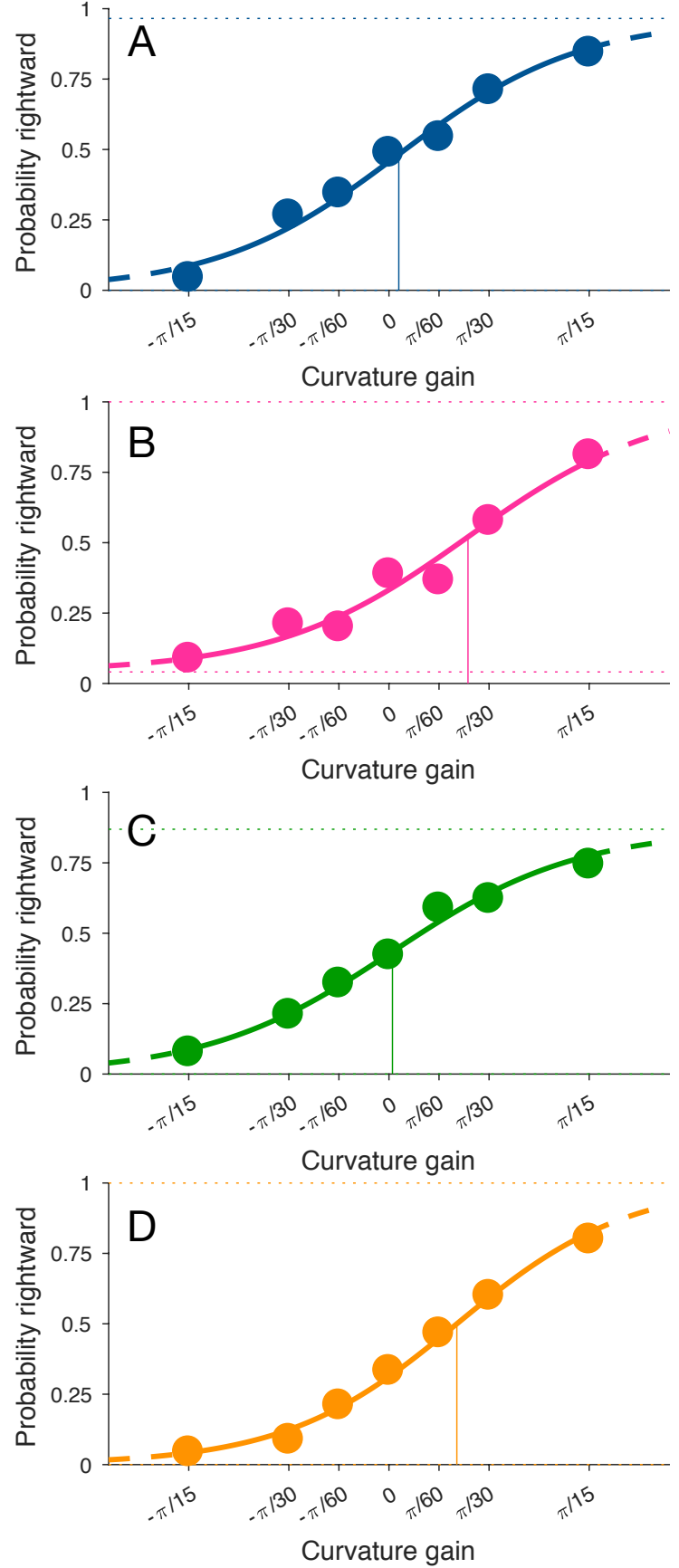

Fig. 5. Psychometric curves for the discrimination of the direction of curvature gain before and after exposure to a constant curvature gain of $G_{C}=+\pi / 30 \mathrm{~m}^{-1}$. Data points are grand averages across participants for the percentage of rightward answers. The thin line marks the pointof-subjective equality (PSE), at which the path appears straight to the participants. A: The psychometric curve in the baseline measurement has a PSE close to zero. B: After adaptation the curve and the PSE are shifted to the right. C: At re-test on the next day the PSE is close to zero again, like in the pre-adaptation baseline in A. D: After further adaptation on day three the shift of the curve is comparable to that after the initial adaptation in B.

between physical and virtual movements. Psychometric measurements of discrimination between leftward and rightward bending paths have been used to estimate detection thresholds, i. e. curvature gains that can be applied imperceptibly. The results of the present study show that these thresholds are plastic and change after prolonged exposure 
to manipulated curvature gain in VR. In particular, application of a constant curvature gain shifts thresholds in the direction of that gain, such that over time stronger curvature gains can be applied without the user noticing. Hence, gain adaptation can be employed in redirected walking to increase its effectiveness and reduce physical space requirements.

In the present experiments, we applied a constant curvature gain throughout the entire adaptation block. This was possible because the user's task in that block was to walk back and forth between two targets. RDW applications with scenarios of this type can directly use a constant curvature gain or at least a constant direction of curvature gain, for example always rightward. Applications include but are not limited to long virtual straight paths or paths, which are curved several times to the same direction. In fact, since many VR scenarios are of a type in which the user first orients herself towards a chosen target and then proceeds straight towards that target, our method might be widely applicable. Most current RDW algorithms are set up to be direction-agnostic, i.e. they steer the user towards the center of the real space using leftward or rightward redirection intermixed (e.g. [10]). However, a one-sided steering algorithm might outperform its twosided equivalent when its minimum turning radius is decreased through adaptation. Such considerations should be taken into account when developing future RDW steering algorithms.

In such scenarios, our findings can be directly applied and the curvature gain can be increased over time to allow stronger redirection. Instead of using a fixed gain to guide users on a circle, a spiral with increasing gains could be used, since the radius of the curves decreases over time. An alternative view is that the the user is first adapted in a limited virtual space, restricted by the physical environment. After adaptation, the virtual space expands while the physical environment remains the same.

One caveat of our approach is that the increase in threshold for the adapted direction comes at the expense of a decrease in threshold of the opposite direction, such that after adaptation of rightward curvatures leftward curvatures become easier to detect. However, in the mentioned scenario in which curvature gain is always in the same direction this should not be a problem and IVEs could be designed specifically to meet such requirements.

In RWD applications that need to change curvature gain direction from trial to trial the situation would likely be different. Adaptation relies on a constant experience of a systematic change in sensorimotor contingencies, which leads to a change in the perceptual normal, i.e. the PSE in the psychometric curve. Thus, one would not expect a change of PSE in a situation of highly fluctuating curvature gains. However, it may be that in such a sensorimotor environment the slope of the psychometric curve may become more shallow as the system may learn to expect an unstable visual feedback. This would also affect thresholds, but it remains to be tested in future work.

On the other hand, adaptation may occur even for applications with variable curvature gain when several trials with constant curvature follow each other, or when a constant curvature gain is applied for some amount of time. This would depend on the speed of adaptation, which could also be tested in future work. Walking adaptation with constant view offset can be observed already after 20 trials [27] and the same might be true for curvature gain adaptation. RDW applications may track the history of curvature gains that were applied to the user and dynamically adjust the range of imperceptible gains that are applied next, based on a dynamic calculation of the user's adaptation state.

When we re-tested the participants' adaptation state on day three we found that adaptation of motor behavior and perceptual thresholds was back to pre-adaptation levels. Our participants had ample exposure to the physical world in which no curvature gain is applied, which likely removed the effect of adaptation. However, from the prism adaptation literature it is known that the brain can store two adaptation states simultaneously and switch between them depending on context cues, such that when wearing one set of prism glasses the brain applies one set of sensorimotor rules while, when wearing a different set it applies a different set of rules [25,38]. The VR setup provides a broad range of characteristics that sets it apart from the normal environment and may act as context cues. These include specific actions like putting on a HMD and specifics of the stimulation like screen resolution, field of view, level of realism or weight of the HMD. Therefore, it may be that prolonged experiences made in the IVE are stored together with these characteristics, like in dual prism adaptation. For example, when looking around with a HMD, users have to make more head rotations than in the real world because the field of view is much smaller, and whenever the user enters the IVE these experiences are available again. Thus, the user learns to use a specific set of sensorimotor rules for specific actions in the IVE and binds them to the VR setup. If gain manipulations applied in the IVE also become part of such a set of learned rules a user that uses the IVE routinely will become used to the gain manipulations and integrate them into the experience. This, in turn, could allow to gradually increase the range of applicable gain manipulations across sessions and, secondly, to use stronger gain manipulations quickly in an experienced user. We aim to test this in future work using repeated adaptation over multiple sessions.

\section{General discussion}

We have shown that adaptation of motor behavior and perception occurs in VR when users are exposed to a constant curvature gain. Such adaptation needs to be taken into account when designing VR environments and when assessing usability and consequences of VR. Potential effects of adaptation on subsequent behavior in the physical world should be considered. In our experiments, no retainment of adaptation was observable in post-testing on the next day but straight walking tasks immediately after adaptation showed deviations in path curvature. While our tests were always confined to the virtual environment, immediate aftereffects may be seen in interactions in the physical environment as well, and should be carefully considered in future work.

The adaptation of perceptual thresholds can be used to improve redirected walking techniques. Curvature gains may be increased over time or may be adjusted dynamically based on the current state of adaptation of the user. The increased manipulation would guide users on a spiral rather than a circle and will eventually decrease the tracking and walking space required for RDW.

Adaptation shifted the detection thresholds but did not change the slope of the psychometric function. Gains around the adapted rightward gain became less perceptible while leftward gains became more noticeable. Whether some form of adaptation may produce a decrease of perceptibility for both directions remains an open question. Perhaps a change in slope may occur if participants were exposed to varying directions and magnitudes of gain manipulation during adaptation. Such parallel adaptation could broaden the psychometric function but it is also possible, that multiple simultaneous adaptation processes hinder each other, or require a much longer adaptation duration.

The threshold measurement that we have obtained may underestimate the magnitude of the adaptive change. For the threshold measurement we needed to expose the participants to a range of gains in both leftward and rightward direction in a randomized order. Since adaptation depends on the exposure to a constant sensorimotor environment a constant curvature gain in our case - the exposure to many different gains in random fluctuation during the threshold task would likely have led to a reduction in adaptation during the measurement, and hence an underestimation of the effect.

\section{Conclusions}

Curvature gains are used in redirected walking to steer users on a predetermined path in physical space while maintaining their immersion of walking in virtual space on a different path. Our experiments showed that users adapt to a constantly applied curvature gain within 20 min and change both their motor behavior and their perception. The adapted curvature gain appears as the new normal state, i. e. the gain that is perceptually equal to straight walking. This adaptation can be used to improve RDW as users can, over time, be exposed to stronger and stronger curvature gains thereby shrinking the physical space requirements for unlimited virtual walking by guiding users on a spiral-like shape curvature. Similar adaptation effects might be possible for other RDW techniques such as rotation, translation and bending gains. 
In the future, we plan to explore the effects of different exposure gains. As described above the considered gain is ecologically valuable, since it is not noticeable by participants and has been used in previous studies $[3,8,14,20]$, however, it might be interesting to explore whether or not different curvature gains could cause the same adaptation effects. In particular, it might be interesting to see how much the detection thresholds and shift of the the PSE can be increased when exposing participants to such gains for a longer period of time, or over successive sessions. Applications are not limited to RDW, but such adaption of motor behaviour might also be used in rehabilitation or training.

Our tests were confined to VR, but immediate aftereffects may be seen in interactions in the real environment as well. While many labs inform user about potential implications of the usage of VR and potential effects to the real word, it is crucial to explore if such adaption even holds in the real world and should be carefully considered in future work.

\section{ACKNOWLEDGMENTS}

This work was supported the German Research Foundation (\# DFG La 952/4-3) and has received funding from the European Unions Horizon 2020 research and innovation programme under the Marie SkodowskaCurie grant agreement No 734227. We thank Steffen Flagge for help with the experiments.

\section{REFERENCES}

[1] M. Azmandian, T. Grechkin, M. Bolas, and E. Suma. Automated path prediction for redirected walking using navigation meshes. In 2016 IEEE Symposium on 3D User Interfaces (3DUI), pp. 63-66, March 2016. doi: 10.1109/3DUI.2016.7460032

[2] B. Bolte and M. Lappe. Subliminal reorientation and reposition in immersive virtual environments using saccadic suppression. IEEE Trans. Vis. Comput. Graph., 21:545 - 552, 2015.

[3] G. Bruder, V. Interrante, L. Phillips, and F. Steinicke. Redirecting walking and driving for natural navigation in immersive virtual environments. IEEE Trans. Vis. Comput. Graph., 18(4):538-545, Apr 2012. doi: 10. 1109/TVCG.2012.55

[4] G. Bruder, F. Steinicke, B. Bolte, P. Wieland, H. Frenz, and M. Lappe Exploiting perceptual limitations and illusions to support walking through virtual environments in confined physical spaces. Displays, 34:132-141, 2013.

[5] H. Bruggeman and W. H. Warren. The direction of walking-but not throwing or kicking-is adapted by optic flow. Psychol. Sci., 21(7):10061013, Jul 2010.

[6] H. Bruggeman, W. Zosh, and W. H. Warren. Optic flow drives human visuo-locomotor adaptation. Curr. Biol., 17(23):2035-2040, 2007.

[7] A. Clark. Whatever next? Predictive brains, situated agents, and the future of cognitive science. Behavioral and Brain Sciences, 36(3):181204, 2013

[8] T. Grechkin, J. Thomas, M. Azmandion, M. Bolas, and E. Suma. Revisiting detection thresholds for redirected walking: combining translation and curvature gains. ACM, 2016. doi: org/10.1145/2931002.2931018

[9] T. A. Herlihey and S. K. Rushton. The role of discrepant retinal motion during walking in the realignment of egocentric space. J. Vis., 12(3), 2012.

[10] E. Hodgson and E. Bachmann. Comparing four approaches to generalized redirected walking: Simulation and live user data. IEEE Trans. Vis. Comput. Graph. (TVCG), 19(4):634-643, 2013.

[11] E. Hodgson, E. Bachmann, and T. Thrash. Performance of redirected walking algorithms in a constrained virtual world. IEEE Trans. Vis. Comput. Graph., 20(4):579-587, April 2014. doi: 10.1109/TVCG.2014.34

[12] E. Hodgson, E. Bachmann, and D. Waller. Redirected walking to explore virtual environments: assessing the potential for spatial interference. ACM Trans. Appl. Percept., 8(4), Nov 2011.

[13] R. S. Kennedy, N. E. Lane, K. S. Berbaum, and M. G. Lilienthal. Simulator sickness questionnaire: An enhanced method for quantifying simulator sickness. Int. J. Aviat. Psychol., 3(3):203-230, 1993.

[14] E. Langbehn, P. Lubos, G. Bruder, and F. Steinicke. Bending the curve: sensitivity to bending of curved paths and application in Room-Scale vr. IEEE Trans. Vis. Comput. Graph., 23(4):1389-1398, 2017. doi: 10. 1109/TVCG.2017.2657220

[15] E. Langbehn, P. Lubos, and Steinicke. Evaluation of locomotion techniques for room-scale vr: Joystick, teleportation, and redirected walking. In Proceedings of the Virtual Reality International Conference (VRIC), 2018.
[16] E. Langbehn and F. Steinicke. Redirected walking in virtual reality. In Springer Encyclopedia of Computer Graphics and Games, pp. 1 - 11, 2018.

[17] E. Langbehn, F. Steinicke, M. Lappe, G. F. Welch, and G. Bruder. In the blink of an eye: leveraging blink-induced suppression for imperceptible position and orientation redirection in virtual reality. ACM Trans. Graph., 37(4):66:1-66:11, 2018.

[18] S. M. Morton and A. J. Bastian. Prism adaptation during walking generalizes to reaching and requires the cerebellum. J. Neurophysiol., 92:24972509, 2004

[19] T. Nescher, Y.-Y. Huang, and A. Kunz. Planing redirection techniques for optimal free walking experience using model predictive control. IEEE 3D User Interfaces, , 2014. doi: 10.1109/3DUI.2014.6798851

[20] C. T. Neth, J. L. Souman, D. Engel, U. Kloos, H. H. Bulthoff, and B. J. Mohler. Velocity-dependent dynamic curvature gain for redirected walking. IEEE Trans. Visual. Comput. Graph. (TVCG), 18(7):1041-1052, 2012. doi: 10.1109/TVCG.2011.275

[21] N. C. Nilsson, T. Peck, G. Bruder, E. Hodgson, S. Serafin, M. Whitton, F. Steinicke, and E. S. Rosenberg. 15 years of research on redirected walking in immersive virtual environments. IEEE Computer Graphics and Applications, 38(2):44 - 56, 2018.

[22] J. K. O'Regan and A. Noe. A sensorimotor account of vision and visual consciousness. Behav. Brain Sci., 24(5):939-1031, 2001.

[23] S. Razzaque, Z. Kohn, and M. Whitton. Redirected walking. In Proceedings of the ACM Eurographics Conference, pp. 289-294, 2001.

[24] S. Razzaque, D. Swapp, M. Slater, M. C. Whitton, and A. Steed. Redirected walking in place. In S. Müller and W. Stüzlinger, eds., ACM International Conference Proceeding Series, vol. 23, pp. 123-130, 2002.

[25] G. M. Redding, Y. Rossetti, and B. Wallace. Applications of prism adaptation: a tutorial in theory and method. Neuroscience and Biobehavioral Reviews, 29:431-444, 2005.

[26] S. Rushton, J. M. Harris, M. R. Lloyd, and J. P. Wann. Guidance of locomotion on foot uses perceived target location rather than optic flow. Curr. Biol., 8:1191-1194, 1998.

[27] J. A. Saunders and F. H. Durgin. Adaptation to conflicting visual and physical heading directions during walking. J. Vis., 11(3), 2011.

[28] H. H. Schütt, S. Harmeling, J. H. Macke, and F. A. Wichmann. Painfree and accurate bayesian estimation of psychometric functions for (potentially) overdispersed data. Vision Research, 122:105-123, 2016.

[29] F. Steinicke, G. Bruder, K. Hinrichs, M. Lappe, S. Kuhl, and P. Willemsen. Judgment of natural perspective projections in head-mounted display environments. In Proceedings of ACM Symposium on Virtual Reality Software and Technology (VRST), pp. 35-42, 2009.

[30] F. Steinicke, G. Bruder, J. Jerald, H. Frenz, and M. Lappe. Estimation of detection thresholds for redirected walking techniques. In IEEE Trans. Vis. Comput. Graph., vol. 16, pp. 17-27, Jan-Feb 2010.

[31] F. Steinicke, Y. Visell, J. Campos, and A. Lecuyer. Human Walking in Virtual Environments: Perception, Technology, and Applications. Springer, 2013.

[32] E. A. Suma, S. Clark, S. Finkelstein, Z. Wartell, M. Krum, and M. Bolas. Leveraging change blindness for redirection in virtual environments. IEEE Virtual Reality, pp. 159-166, Mar 2011.

[33] E. A. Suma, Z. Lipps, S. Finkelstein, D. M. Krum, and M. Bolas. Impossible spaces: maximizing natural walking in virtual environments with self-overlapping architecture. IEEE Trans. Vis. Comput. Graph., 18(4):555-564, 2012. doi: 10.1109/TVCG.2012.47

[34] Q. Sun, A. Patney, L.-Y. Wei, O. Shapora, J. Li, P. Asente, S. Zhu, M. McGuire, D. Luebke, and A. Kaufmann. Towards virtual reality infinite walking: dynamic saccadic redirection. ACM, 37(4), 2018.

[35] Q. Sun, L.-Y. Wei, and A. Kaufmann. Mapping virtual and physical reality. ACM, 2016. doi: org/10.1145/2897824.2925883

[36] M. Usoh, K. Arthur, M. C. Whitton, R. Bastos, A. Steed, M. Slater, and F. P. J. Brooks. Walking $>$ walking-in-place $>$ flying, in virtual environments. In Proceedings of the 26th Annual Conference on Computer Graphics and Interactive Techniques, pp. 359-364, 1999.

[37] M. Usoh, E. Catena, S. Arman, and M. Slater. Using presence questionnaires in reality. Presence: Teleoperators and Virtual Environments, 9:497-503, 2000.

[38] R. B. Welch, B. Bridgeman, S. Anand, and K. E. Browman. Alternating prism exposure causes dual adaptation and generalization to a novel displacement. Percept. Psychophys., 54(2):195-204, 1993. 